\title{
Estimating the quality of $3 D$ protein models using the ModFOLD7 server
}

Book or Report Section

Accepted Version

Maghrabi, A. H. A. and McGuffin, L. J. ORCID:

https://orcid.org/0000-0003-4501-4767 (2020) Estimating the quality of 3D protein models using the ModFOLD7 server. In:

Daisuke, K. (ed.) Protein Structure Prediction. Methods in Molecular Biology, 2165. Springer, pp. 69-81. ISBN 978-10716-0708-4 doi: https://doi.org/10.1007/978-1-0716-0708-4_4 (Protein Structure Prediction) Available at https://centaur.reading.ac.uk/90370/

It is advisable to refer to the publisher's version if you intend to cite from the work. See Guidance on citing.

To link to this article DOI: http://dx.doi.org/10.1007/978-1-0716-0708-4_4

Publisher: Springer

All outputs in CentAUR are protected by Intellectual Property Rights law, including copyright law. Copyright and IPR is retained by the creators or other copyright holders. Terms and conditions for use of this material are defined in the End User Agreement. 


\section{CentAUR}

Central Archive at the University of Reading

Reading's research outputs online 


\title{
Author's Proof
}

\section{Chapter 4}

\section{Estimating the Quality of 3D Protein Models Using the ModFOLD7 Server}

\author{
Ali H. A. Maghrabi and Liam J. McGuffin
}

\begin{abstract}
Assessing the accuracy of 3D models has become a keystone in the protein structure prediction field. 6 ModFOLD7 is our leading resource for Estimates of Model Accuracy (EMA), which has been upgraded by 7 integrating a number of the pioneering pure-single- and quasi-single-model approaches. Such an integra- 8 tion has given our latest version the strengths to accurately score and rank predicted models, with higher 9 consistency compared to older EMA methods. Additionally, the server provides three options for producing 10 global score estimates, depending on the requirements of the user: (1) ModFOLD7_rank, which is 11 optimized for ranking/selection, (2) ModFOLD7_cor, which is optimized for correlations of predicted 12 and observed scores, and (3) ModFOLD7 global for balanced performance. ModFOLD7 has been ranked 13 among the top few EMA methods according to independent blind testing by the CASPI3 assessors. 14 Another evaluation resource for ModFOLD7 is the CAMEO project, where the method is continuously 15 automatically evaluated, showing a significant improvement compared to our previous versions. The 16 ModFOLD7 server is freely available at http://www.reading.ac.uk/bioinf/ModFOLD/.

Key words Estimates of model accuracy (EMA), Model quality assessment (MQA), Protein structure 18 prediction, Protein modeling, Tertiary structure prediction, Critical assessment of techniques for 19 protein structure prediction (CASP), Continuously evaluate the accuracy and reliability of predictions 20 (CAMEO)

1 Introduction

Since researchers from different fields of biological sciences started 23 relying on the three-dimensional structural models of proteins, 24 prediction programs have been improving rapidly. One of the 25 major components of structure prediction pipelines is the evalua- 26 tion or assessment of the predicted model accuracy. It is possible to 27 generate many hundreds of alternative $3 \mathrm{D}$ models for any give 28 protein target using many different algorithms. Often, the best 29 modeling method is not always the most accurate for a given target, 30 so it is problematic to choose rank and select the models that are 31 most likely to be the closest to the native structure. Furthermore, 32 local regions of models may differ in quality, and so it may help a 33 


\section{Author's Proof}

biologist to know whether their specific regions of interest are accurately modeled, for example, predicted interface/interacting residues. Such problems have been recognized by the field of structural bioinformatics, and many developers have focused their attention toward improving methods for Model Quality Assessment (QA) that support their prediction pipelines. Such tools and servers are also currently referred to as the Estimates of Model Accuracy (EMA) methods.

The EMA (a.k.a. QA) methods and servers were included for evaluation as a category in two major worldwide organizations that are specialized in the protein structure prediction field. The first organization conducts independent blind testing with the Critical Assessment of Techniques for Protein Structure Prediction (CASP) [1] experiments, which are held every other year. The second organization is the continuously automatic model evaluation project called CAMEO [2]. Both organizations have highlighted the importance of the EMA development for the improvement of protein structure prediction and have helped to encourage progress in the field.

Modern methods of EMA can be classified into three broad categories. (1) The pure-single-model methods, which can score the data from the information of an individual model-they are featured by their rapid processing and their strong performance at model ranking and selection, but they often produce less consistent global scores. (2) The clustering/consensus approaches, which use multiple alternative models build for the same protein targetthese types of methods have the opposite features of the singlemodel methods, and they have been far more accurate but are more computationally intensive and do not work when very few similar models are available. (3) The quasi-single-model methods, which can score an individual model against a pool of reference alternative models that are generated from the same target sequence. Quasisingle-model methods attempt to provide comparable accuracy to clustering methods, while addressing real-life needs of researchers with few/single models.

ModFOLD [3] is our EMA protocol, and various successive versions have been competing with the top-leading model quality assessment programs throughout the past 10 years. ModFOLD was built in the beginning as two separate methods. The original singlemodel method was called by its own original name, ModFOLD. Additionally, we developed a clustering-based method, called ModFOLDclust [4]. Over the years, both methods have been merged with the adoption of a number of other methods to develop a new ModFOLD program which was a pioneer of the quasi-singlemodel approach.

The quasi-single-model approach was firstly implemented with the third version of ModFOLD [5]. By using this approach, ModFOLD3 was able to generate reference sets of models from the 
target sequence, using the IntFOLD-TS [6] method, which were 82 used for comparison with the submitted model using ModFOLD- 83 clust2 [4]. ModFOLD has since undergone a number of updates 84 through versions 4 [7], 5 [8], and 6 [9], which have maintained the 85 use of a quasi-single-model approach. Each successive version has 86 been ranked among the top-performing EMA methods of the 87 recent CASP experiments. The implementation of quasi-single 88 method has helped our ModFOLD pipeline keep its competitive- 89 ness using the predictive power offered by clustering-based meth- 90 ods, as well as being capable of making predictions for a single 91 model at a time. While we have made significant progress in perfor- 92 mance over the years with our ModFOLD methods, there is still 93 room for improvement in many aspects of EMA. 94

Here, we describe significant major updates to the ModFOLD 95 server. The server has been popular with modelers around the 96 world, having completed hundreds of thousands of EMA jobs for 97 thousands of unique users over the past decade.

\title{
然
}

.

\section{(1)}




\section{Author's Proof}

Ali H. A. Maghrabi and Liam J. McGuffin

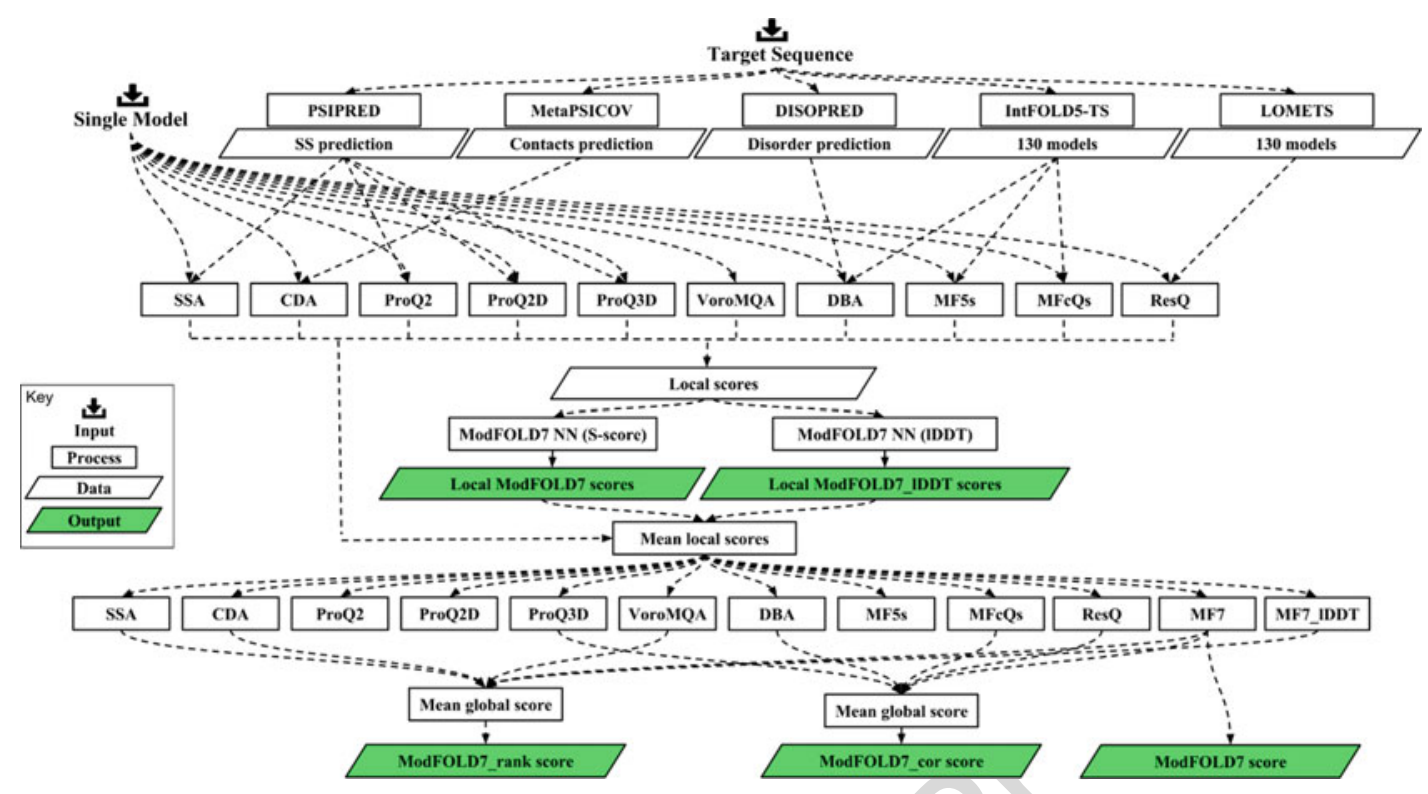

Fig. 1 Flow of data illustrating the local and global estimates of model accuracy in ModFOLD7. The method pipeline starts with two inputs, the target sequence and a single model. The target sequence is evaluated with five preprocessing methods. The resulting data from the preprocessing methods with the input single model then are evaluated with ten scoring methods resulting in local score input data. Next, the local scores are processed using two neural networks (NN) trained to two target functions, the $S$-score and the IDDT score, resulting in the final local score outputs. Lastly, the mean local scores from each method are used to form 12 global scores, which are then optimally combined in the different ways indicated to form the three variants of ModFOLD7

\subsection{The ModFOLD7 Component Per- Residue/Local Quality Scoring Methods}

The ModFOLD7 NNs were trained using two separate target functions for each residue in a model: the residue contact-based IDDT score and the superposition-based $S$-score which has been used in previous versions of ModFOLD. The RSNNS package for $\mathrm{R}$ was used to construct the NNs, which were trained using data derived from the evaluation of CASP11 and 12 server models versus native structures. The per-residue similarity scores were calculated using a simple multilayer perceptron (MLP). For the method trained using the IDDT score (ModFOLD7_res_lddt), the MLP input consisted of a sliding window $($ size $=5$ ) of per-residue scores from all ten of the methods described above, and the output was a single quality score for each residue in the model (50 inputs, 25 hidden, 1 output). For the method trained using the $S$-score (ModFOLD7_res), this time only seven of the ten methods were used as inputs-all apart from the ProQ2, CDA, and SSA scores-with a sliding window ( size $=5$ ), therefore 35 inputs, 18 hidden, 1 output. For both of the per-residue scoring methods, the similarity scores, $s$, for each residue were converted back to distances, $d$, with $d=3.5 \sqrt{ }((1 / s)-1)$. 


\section{Author's Proof}

\subsection{The ModFOLD7 Global Scoring Methods}

2.3 Server Inputs and Outputs
Global scores were calculated by taking the mean per-residue scores 148 (the sum of the per-residue similarity scores divided by sequence 149 lengths) for each of the ten individual component methods, 150 described above, plus the NN output from ModFOLD7_res and 151 ModFOLD7_res_lddt. Furthermore, three additional quasi-single 152 global model quality scores were generated for each model based 153 on the original ModFOLDclust, ModFOLDclustQ, and Mod- 154 FOLDclust 2 global scoring methods (in a similar vein to the Mod- 155 FOLD4_single and ModFOLD5_single global scores, tested in 156 CASP10 and CASP11, respectively). Thus, we ended up with 157 15 alternative global QA scores, which could be combined in 158 various ways in order to optimize for the different facets of the 159 quality estimation problem. For the CASP13 experiment, we 160 registered three ModFOLD7 global scoring variants: (1) The 161 ModFOLD7 global score, which used the mean per-residue NN 162 output score from ModFOLD7_res-this score considered alone 163 was found to have a good balance of performance both for correla- 164 tions of predicted versus observed scores and rankings of the 165 top models. (2) The ModFOLD7_cor global score variant 166 $((M F c Q s+D B A+\operatorname{ProQ} 3 D+\operatorname{Res} Q+$ ModFOLD7_res $) / 5)$ was 167 found to be an optimal combination for producing good correla- 168 tions with the observed scores, that is, the predicted global quality 169 scores produced should produce closer to linear correlations with 170 the observed global quality scores. (3) The ModFOLD7_rank 171 global score variant $((C D A+S S A+$ VoroMQA + Mod- 172 FOLD7_res + ModFOLD7res_LDDT)/5) was found to be an optimal 173 combination for ranking, that is, the top-ranked models (top 1) 174 should be closer to the highest accuracy, but the relationship 175 between predicted and observed scores may not be linear. The 176 local scores of the ModFOLD7 and ModFOLD_rank variants 177 used the output from the ModFOLD7_res NN, whereas the 178 ModFOLD_cor variant used the local scores from the 179 ModFOLD7_res_lddt NN. 180

Like the previous versions, the ModFOLD7 server requires only 182 the amino acid sequence for the protein target and a single 3D 183 model (in PDB format) for evaluation. However, users can upload 184 more than one PDB file in a compressed archive. Optionally, users 185 can also give their target a name and also provide their e-mail 186 address, so that they can receive a notification of the result (see 187 Notes 1-6).

The results are provided in a clean and simple user interface so 189 that it can be interpreted easily by nonexperts at a glance. Once the 190 prediction process is complete, a results page is generated contain- 191 ing a single table summarizing the quality assessment scores for 192 each submitted model. Each assessed model is represented in the 193 table graphically, with thumbnail images of the local error plots and 194 annotated 3D models. Images in the table are clickable for detailed 195 


\section{Author's Proof}

Ali H. A. Maghrabi and Liam J. McGuffin

\subsection{Independent Benchmarking and Cross-Validation}

3D visualization using the JSmol/HTML5 framework. Conveniently, interactive 3D results can also be viewed on mobile devices without any plugin requirement. The results table shows a global score for each model, a $p$-value indicating the likelihood that the model is incorrectly folded and a plot of the local errors in the model in Ångströms. Users can also download the models annotated with the ModFOLD7 predicted local quality scores, which have been inserted into the $B$-factor column of the ATOM records for each submitted model. The raw machine-readable data files for each set of predictions, which comply with the CASP data standards, are also provided for developers and more advanced users. An overview of the ModFOLD7 interface is shown in Fig. 2 (see Notes 7-12).

The three alternative optimized scoring methods of the ModFOLD7 server have been benchmarked against their respective previous versions from the ModFOLD6 server (Fig. 3). For the cumulative GDT_TS of top-ranked model, ModFOLD6_rank method was giving a score below 44.5 as their highest, whereas ModFOLD7_rank was able to cross the 45 and go higher. For the Pearson correlation comparing the predicted score versus the observed score (GDT_TS), ModFOLD6_cor achieved a correlation 0.9250, while for ModFOLD7_cor, the correlation was found to be over 0.9300 . For the evaluation of local model quality prediction accuracy using the area under the ROC curve (AUC) (where residues with IDDT scores $\leq 0.6=0$ ), ModFOLD6 could not reach an AUC score of 0.93, whereas ModFOLD7 was closer to 0.95 . Such results indicate that our latest version, ModFOLD7, has demonstrated progress in performance compared to ModFOLD6, and according to many measures, the improvements are significant.

ModFOLD7 is also one of the EMA servers that are continuously independently benchmarked for local EMA performance by the evaluating organization, CAMEO. For the last year, the

In 2018, the ModFOLD7 servers participated in the latest world- 


\section{Author's Proof}

\section{Input page}

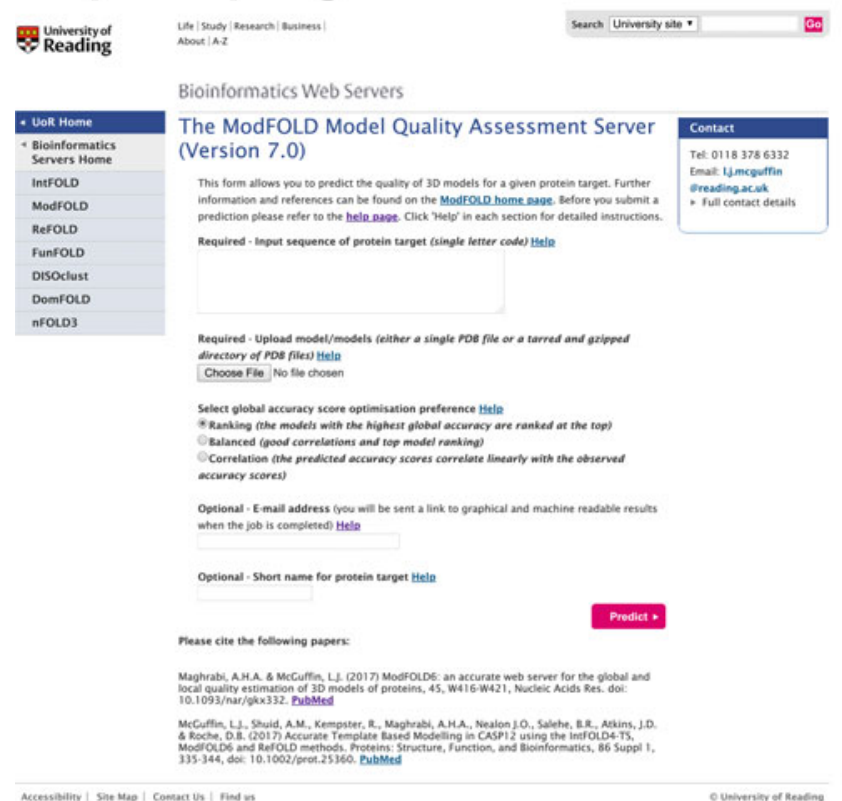

\section{Output page}

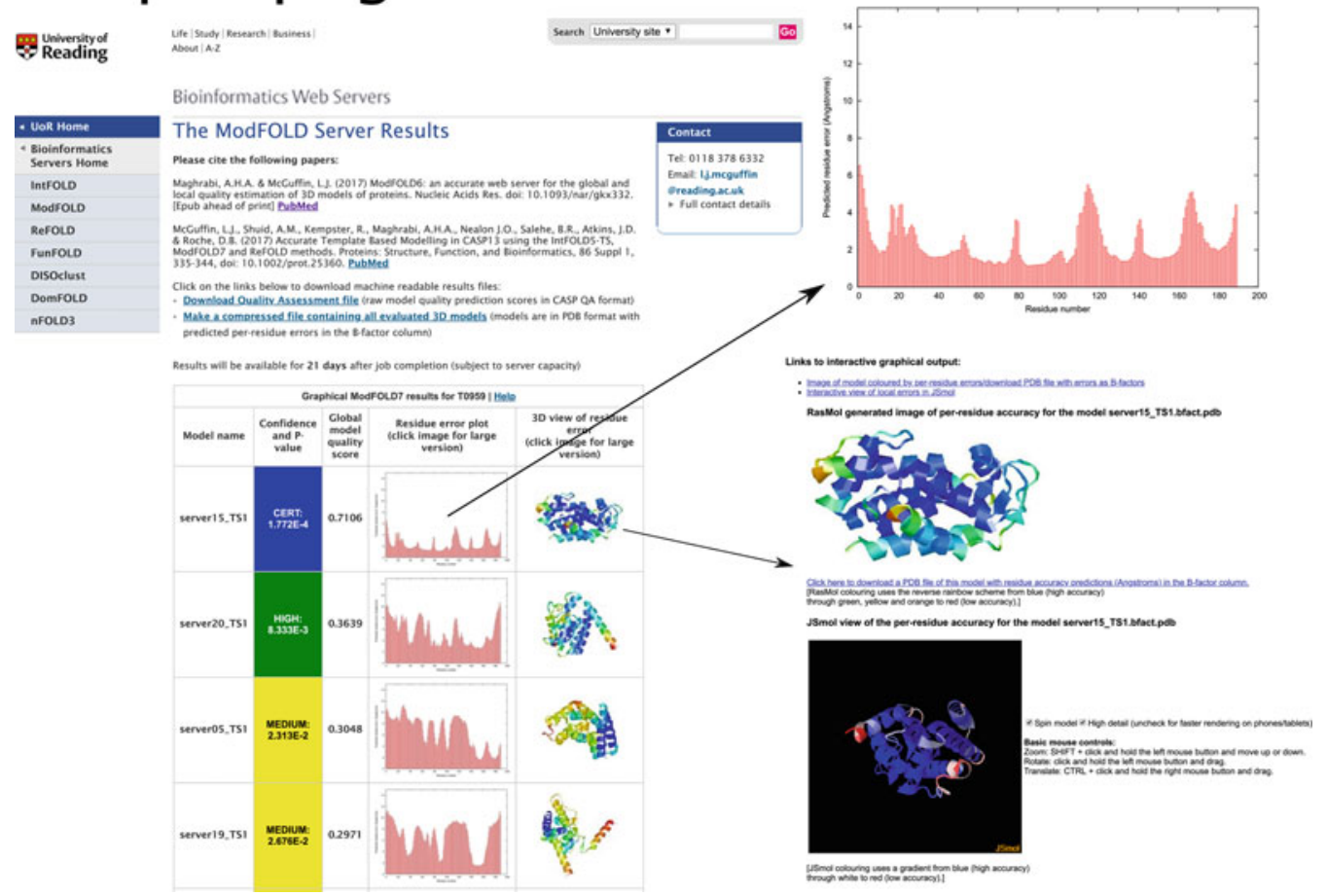

Fig. 2 ModFOLD7 server inputs and outputs pages. Inputs page: containing a text box to paste the amino acid sequence of protein target in single-letter code, a push button to upload model/models (either a single PDB file or a tarred and gzipped directory of PDB files) of the protein target, three options to select the global accuracy score optimization preference, and two optional text boxes to input the user e-mail address and to give a short name for protein target. Outputs page: showing the result page for models submitted to CASP13 generated for target T0959. The main output page is shown with summary tables of the results for each model. Results can also be visualized in more detail by clicking on the thumbnail images in the main table 


\section{Author's Proof}

Ali H. A. Maghrabi and Liam J. McGuffin
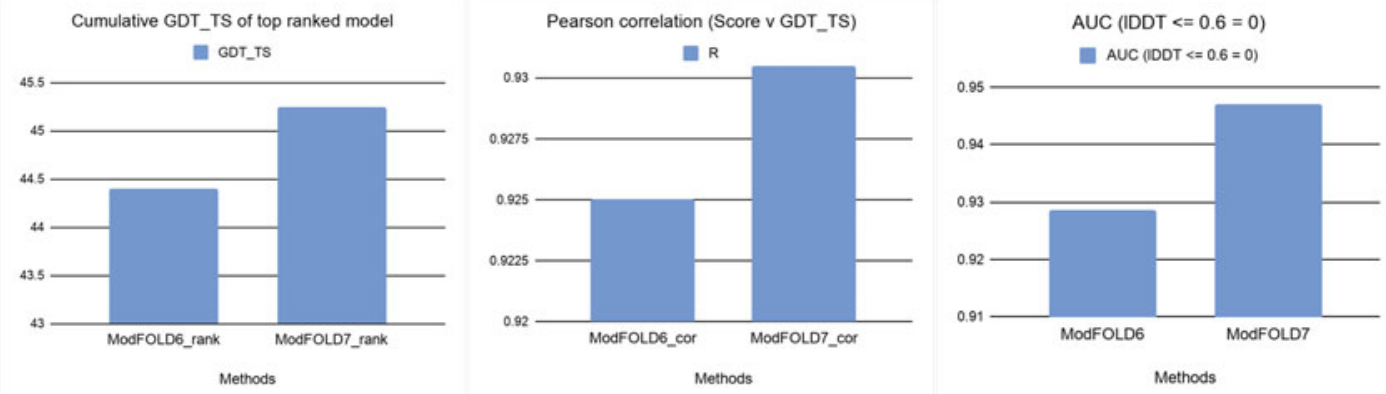

Fig. 3 Histograms showing a comparison between the three variants of ModFOLD6 and the respective variants of ModFOLD7 using three evaluation methods: the cumulative GDT_TS of top-ranked models, the Pearson correlations between predictive and observed scores, and the local accuracy as measured by the AUC score $($ IDDT $\leq 0.6=0)$. Evaluation is based on cross-validated CASP11 data

Table 1

\section{Top EMA methods in CAMEO}

\begin{tabular}{|c|c|c|c|c|c|c|c|c|c|c|c|}
\hline \multirow[b]{2}{*}{ Server } & \multicolumn{3}{|c|}{ Structural models } & \multicolumn{2}{|l|}{ ROC } & \multicolumn{2}{|c|}{$\begin{array}{l}\text { ROC } \\
\text { normalized }\end{array}$} & \multicolumn{2}{|l|}{ PR } & \multicolumn{2}{|c|}{$\begin{array}{l}\text { PR } \\
\text { normalized }\end{array}$} \\
\hline & Submitted & Received & $\%$ & $\begin{array}{l}\text { AUC } \\
0,1\end{array}$ & $\begin{array}{l}\text { AUC } \\
0,0.2\end{array}$ & $\begin{array}{l}\text { AUC } \\
0,1\end{array}$ & $\begin{array}{l}\text { AUC } \\
0,0.2\end{array}$ & $\begin{array}{l}\text { AUC } \\
0,1\end{array}$ & $\begin{array}{l}\text { AUC } \\
0.8,1\end{array}$ & $\begin{array}{l}\text { AUC } \\
0,1\end{array}$ & $\begin{array}{l}\text { AUC } \\
0.8,1\end{array}$ \\
\hline QMEANDisCo & 9816 & 9041 & 92.1 & 0.93 & 0.77 & 0.86 & 0.71 & 0.9 & 0.66 & 0.83 & 0.61 \\
\hline ModFOLD7_IDDT & 9816 & 8283 & 84.4 & 0.91 & 0.71 & 0.77 & 0.6 & 0.87 & 0.61 & 0.74 & 0.51 \\
\hline ModFOLD6 & 9816 & 6709 & 68.3 & 0.89 & 0.65 & 0.61 & 0.44 & 0.84 & 0.58 & 0.57 & 0.4 \\
\hline QMEAN & 9816 & 9054 & 92.2 & 0.87 & 0.61 & 0.8 & 0.56 & 0.81 & 0.53 & 0.74 & 0.49 \\
\hline ProQ2 & 9816 & 9464 & 96.4 & 0.86 & 0.58 & 0.82 & 0.56 & 0.79 & 0.5 & 0.76 & 0.48 \\
\hline ModFOLD4 & 9816 & 7191 & 73.3 & 0.85 & 0.57 & 0.62 & 0.42 & 0.78 & 0.49 & 0.57 & 0.36 \\
\hline
\end{tabular}

One year of data downloaded from http://www.cameo3d.org/. One year [2018-03-30-2019-03-23]_“All” dataset. The table is sorted by the ROC AUC score

$R O C$ receiver operating characteristic, $A U C$ area under the ROC curve, $P R$ precision and recall

sequence by testing them objectively via the process of blind prediction. The competition includes many subcategories, one of them is the Estimate of Model Accuracy (EMA) where our ModFOLD7 methods are independently evaluated. The CASP assessors provide sequences of proteins whose structures have never been observed before. Participants use their prediction servers in order to generate the 3D models of the target structures. Once server models have been generated for a given target, they are then used for the EMA in order to estimate the accuracy of the predicted models for each 


\section{Author's Proof}

In CASP13, the assessors provide predictors with anonymous 253 protein sequence (targets), and these targets are submitted by 254 different biological research teams around the world who have a 255 vested interest in determining their structures. An example of one 256 of these protein targets is Endolysin KPP12 (CASP3 target 257 T0962), a bacteriophage found to have a therapeutic effect in 258 Pseudomonas aeruginosa keratitis [18]. The study shows that the 259 morphological and DNA sequence analysis of KPP12 have led to 260 identifying the family of that protein and the similarities with other 261 viruses, and therefore, researchers are testing whether the protein is 262 the same as its family members. Using KPPl2 as a treatment can 263 result in the suppression of neutrophil infiltration, and it also can 264 greatly enhance bacterial clearance in the infected cornea. 265

The only available data for KPPl2 were the sequence. Partici- 266 pants from different organizations and companies started to predict 267 the structure of that protein by using their own methods. After 268 structure prediction, the created models were assessed in terms of 269 its quality and how close are these models to their protein native 270 structures. The results showed that ModFOLD7 has given the best 271 EMA score among all the other methods in all measurements such 272 as LDDT with 0.660 and CAD with 1.990 (Table 2). Such infor- 273 mation about model quality is invaluable in identifying: firstly, the 274 very best $3 \mathrm{D}$ models of a protein that are the closest to the native 275

Table 2

The top ten EMA methods for Target T0962 (KPP12) in CASP13 in terms of absolute differences in score between the top selected model and the best model according to observed structure (smaller scores indicate higher performing methods)

\begin{tabular}{|c|c|c|c|c|c|}
\hline Rank & Gr. Name & GDT_TS & LDDT & CAD(AA) & SG \\
\hline 1 & SBROD-plus & 0.000 & 0.660 & 1.990 & 0.000 \\
\hline 2 & ModFOLD7 & 0.000 & 0.660 & 1.990 & 0.000 \\
\hline 3 & ModFOLD7_cor & 0.000 & 0.660 & 1.990 & 0.000 \\
\hline 4 & MASS2 & 10.170 & 2.110 & 3.991 & 8.475 \\
\hline 5 & Bhattacharya-Server & 10.170 & 2.110 & 3.991 & 8.475 \\
\hline 6 & Pcons & 6.215 & 2.660 & 3.121 & 10.452 \\
\hline 7 & VoroMQA-B & 4.802 & 2.850 & 2.033 & 5.933 \\
\hline 8 & Kiharalab & 4.802 & 2.850 & 2.033 & 5.933 \\
\hline 9 & ProQ4 & 4.802 & 2.850 & 2.033 & 5.933 \\
\hline 10 & MASS1 & 4.802 & 2.850 & 2.033 & 5.933 \\
\hline
\end{tabular}

EMA methods are evaluated for target T0962 in CASP13. The evaluation was performed using GDT_TS, IDDT, CAD, t.13 and SG measuring scores. Only the top ten methods are shown, and the table is sorted using IDDT scores. The scores are calculated over all models for all targets (QA stage 2-best 150). The data are downloaded from http://predictioncenter. org/caspl3/qa_diff2best.cgi 


\section{Author's Proof}

Ali H. A. Maghrabi and Liam J. McGuffin

structures, secondly, the likelihood that models are of good or poor quality overall, and finally, the magnitude of errors in specific local regions of the protein and the regions that are likely to have the fewest errors.

1. The ModFOLD server version 7.0 requires the amino acid sequence of your target protein and either a single $3 \mathrm{D}$ model file in PDB format or a tarball containing a directory of multiple separate files in PDB format. To produce a tarball file for your own 3D models, for Linux/OSX/other Unix users: (a) Tar up the directory containing your PDB files, for example, type the following at the command line: tar cvf my_models.tar my_models/, (b) Gzip the tar file, for example, gzip my_models.tar, (c) upload the gzipped tar file (e.g., my_models.tar.gz) to the ModFOLD server; and for Windows users: (a) download a file archiver application such as 7-zip, (b) select the directory (folder) of model files to add to the .tar file, click "Add," select the "tar" option as the "Archive format:", and save the file as something memorable, for example, my_models.tar, (c) select the tar file, click "Add," and then select the "GZip" option as the "Archive format:" - the file should then be saved as my_models.tar.gz, and (d) upload the gzipped tar file (e.g., my_models.tar.gz) to the ModFOLD server.

2. Providing the e-mail address will give the permission to send a link with the graphical results and machine-readable results directly after the predictions are completed. However, if the user does not provide the e-mail address, then she/he must bookmark the results page in order to view and refer to it when it is available.

3. In the text box labeled "Input sequence of protein target," users should carefully paste in the full amino acid for the interested target protein in single-letter format. An example sequence (CASP13 target T0949) is inserted as MAAKKGMTTVLVSAVICAGVIIGALQWEKAVALPNPSG QVINGVHHYTIDEFNYYYKPDRMTWHVGEKVELTIDN RSQSAPPIAHQFSIGRTLVSRDNGFPKSQAIAVGWKDNF FDGVPITSGGQTGPVPAFSVSLNGGQKYTFSFVVPNKPG KWEYGCFLQTGQHFMNGMHGILDILPAQGS.

4. It is important that the user provides the full sequence that 314 corresponds to the sequence of residue coordinates in the model file. If the model does not contain numbering which corresponds directly to the order of residues in the sequence file, then the server will attempt to renumber the residues in the model files accordingly. However, submitting a model file 
with residues that are not contained in the provided sequence 320 will not complete the prediction for that model.

5. Users must ensure that each PDB file contains the coordinates 322 for one model only. Please do not upload a single PDB file 323 containing the coordinates for multiple alternative NMR mod- 324 els. The coordinates for multiple models should always be 325 uploaded as a tarred and gzipped directory of separate files. $\quad 326$

6. Assigning a short memorable name to user's prediction jobs is 327 useful for identifying and distinguishing them, because Mod- 328 FOLD will not necessarily return the results in the order the 329 user submitted them.

7. The results table is ranked according to decreasing global 331 model quality score. The global model quality scores range 332 between 0 and 1 . In general, scores less than 0.2 indicate that 333 there may be incorrectly modeled domains, and scores greater 334 than 0.4 generally indicate more complete and confident mod- 335 els, which are highly similar to the native structure. If the global 336 model quality scores are low, then the per-residue scores can 337 give you an idea of specific domains or regions in your protein 338 that might be correctly modeled.

8 . From the global scores, the $p$-value which represents the prob- 340 ability that each model is incorrect can be calculated. In other 341 words, for a given predicted model quality score, the $p$-value is 342 the proportion of models with that score that do not share any 343 similarity with the native structure $(\mathrm{TM}$-score $<0.2)$. Each 344 model is also assigned a color-coded confidence level depend- 345 ing on the $p$-value: $p<0.001=$ blue $=$ CERT $=$ Less than a 346 $1 / 1000$ chance that the model is incorrect, 347 $p<0.01=$ green $=\mathrm{HIGH}=$ Less than a $1 / 100$ chance that 348 the model is incorrect, $p<0.05=$ yellow $=$ MEDIUM $=$ Less 349 than a $1 / 20$ chance that the model is incorrect, 350 $p<0.1=$ orange $=\mathrm{LOW}=$ Less than a $1 / 10$ chance that 351 the model is incorrect, $p>0.1=\mathrm{red}=\mathrm{POOR}=$ Likely to be a 352 poor model with little or no similarity to the native structure. 353

9. The per-residue scores indicate the predicted distance 354 (in Angstroms) between the CA atom of the residue in the 355 model and the $\mathrm{CA}$ atom of the equivalent residue in the native 356 structure. Thumbnail images of plots depicting the per-residue 357 error versus residue number are included in each row in the 358 results table. Each of the thumbnails links to a page that dis- 359 plays a larger view of the plot and contains a further link to 360 download a PostScript version. Each row in the table also dis- 361 plays a thumbnail of the $3 \mathrm{D}$ cartoon view of the model which is 362 color coded with the residue error according to the RasMol 363 temperature coloring scheme. Each small image also links to a 364 page that shows a larger image of the $3 \mathrm{D}$ view and contains a 365 


\section{Author's Proof}

Ali H. A. Maghrabi and Liam J. McGuffin

link to download a PDB file of the model with residue accuracy predictions (Angstroms) in the $B$-factor column. The model is also loaded into JSmol for convenient interactive viewing of per-residue errors within the browser.

10. The time taken for a prediction will depend on the length of sequence, the number of models submitted, and the load on the server. For a new run on single model, the user should typically receive his/her results back within $24 \mathrm{~h}$, once the job is running. Large batches of models (several hundred) for a single target may take several days to process. If the user has already submitted a model for the same target sequence within the same week, then the reference model library for that sequence will already be available to the server (the results will be cached) and so she/he will receive the results back much more quickly (within a few hours).

11. For fair usage policy, the users are allowed to have one job running at a time for each IP address, so please wait until your previous job completes before submitting further data. If you already have a job running, then you will be notified, and your uploaded data will be deleted. Once your job has completed, your IP address will be unlocked and you will be able to submit new data.

12. Users should check the header of the machine-readable results file (provided as a link at the top of the result page) for any errors that may have occurred following file submission. Please e-mail us for help if you encounter a persistent error.

1. Moult J, Fidelis K, Kryshtafovych A, Schwede T, Tramontano A (2014) Critical assessment of methods of protein structure prediction (CASP)-round $x$. Proteins 82 (Suppl 2):1-6. https://doi.org/10.1002/ prot. 24452

2. Haas J, Barbato A, Behringer D, Studer G, Roth S, Bertoni M, Mostaguir K, Gumienny R, Schwede T (2018) Continuous Automated Model EvaluatiOn (CAMEO) complementing the critical assessment of structure prediction in CASP12. Proteins 86:387-398. https://doi.org/10.1002/prot. 25431

3. McGuffin LJ (2007) Benchmarking consensus model quality assessment for protein fold recognition. BMC Bioinformatics 8:345. https:// doi.org/10.1186/1471-2105-8-345

4. McGuffin LJ, Roche DB (2010) Rapid model quality assessment for protein structure predictions using the comparison of multiple models without structural alignments. Bioinformatics 26:182-188. https://doi.org/10.1093/bioin formatics/btp629

5. Roche DB, Buenavista MT, McGuffin LJ (2014) Assessing the quality of modelled 3D protein structures using the ModFOLD server. In: Kihara D (ed) Protein structure prediction. Springer, New York, pp 83-103

6. McGuffin LJ, Roche DB (2011) Automated tertiary structure prediction with accurate local model quality assessment using the intfold-ts method. Proteins 79:137-146. https://doi.org/10.1002/prot.23120

7. McGuffin LJ, Buenavista MT, Roche DB (2013) The ModFOLD4 server for the quality assessment of 3D protein models. Nucleic Acids Res 41:W368-W372. https://doi.org/ $10.1093 / \mathrm{nar} / \mathrm{gkt} 294$

8. McGuffin LJ, Atkins JD, Salehe BR, Shuid AN, Roche DB (2015) IntFOLD: an integrated server for modelling protein structures and 


\section{Author's Proof}

functions from amino acid sequences. Nucleic Acids Res 43:W169-W173. https://doi.org/ $10.1093 / \mathrm{nar} / \mathrm{gkv} 236$

9. Maghrabi AHA, McGuffin LJ (2017) ModFOLD6: an accurate web server for the global and local quality estimation of $3 \mathrm{D}$ protein models. Nucleic Acids Res 45:W416-W421. https://doi.org/10.1093/nar/gkx332

10. Jones DT, Singh T, Kosciolek T, Tetchner $S$ (2015) MetaPSICOV: combining coevolution methods for accurate prediction of contacts and long range hydrogen bonding in proteins. Bioinformatics 31:999-1006. https://doi. org/10.1093/bioinformatics/btu791

11. Buchan DWA, Minneci F, Nugent TCO, Bryson K, Jones DT (2013) Scalable web services for the PSIPRED protein analysis workbench. Nucleic Acids Res 41:W349-W357. https://doi.org/10.1093/nar/gkt381

12. Uziela K, Wallner B (2016) ProQ2: estimation of model accuracy implemented in Rosetta. Bioinformatics 32:1411-1413. https://doi. org/10.1093/bioinformatics/btv767

13. Uziela K, Hurtado DM, Wallner B, Elofsson A (2016) ProQ3D: improved model quality assessments using Deep Learning. ArXiv161005189 Q-Bio

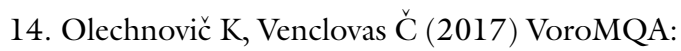
assessment of protein structure quality using interatomic contact areas. Proteins 85:1131-1145. https://doi.org/10.1002/ prot. 25278

15. Jones DT, Cozzetto D (2015) DISOPRED3: precise disordered region predictions with annotated protein-binding activity. Bioinformatics 31:857-863. https://doi.org/10. 1093/bioinformatics/btu744

16. Yang J, Wang Y, Zhang Y (2016) ResQ: an approach to unified estimation of $\mathrm{B}$-factor and residue-specific error in protein structure prediction. J Mol Biol 428:693-701. https://doi. org/10.1016/j.jmb.2015.09.024

17. Wu S, Zhang Y (2007) LOMETS: a local metathreading-server for protein structure prediction. Nucleic Acids Res 35:3375-3382. https://doi.org/10.1093/nar/gkm251

18. Fukuda K, Ishida W, Uchiyama J, Rashel M, Kato S, Morita T, Muraoka A, Sumi T, Matsuzaki S, Daibata M, Fukushima A (2012) Pseudomonas aeruginosa keratitis in mice: effects of topical bacteriophage KPPI2 administration. PLoS One 7:e47742. https://doi. org/10.1371/journal.pone.0047742 